\title{
Active Learning in Partially Observable Markov Decision Processes
}

\author{
Robin Jaulmes, Joelle Pineau, and Doina Precup \\ McGill University, School of Computer Science, \\ 3480 University St., Montreal, QC, H3A2A7
}

\begin{abstract}
This paper examines the problem of finding an optimal policy for a Partially Observable Markov Decision Process (POMDP) when the model is not known or is only poorly specified. We propose two approaches to this problem. The first relies on a model of the uncertainty that is added directly into the POMDP planning problem. This has theoretical guarantees, but is impractical when many of the parameters are uncertain. The second, called MEDUSA, incrementally improves the POMDP model using selected queries, while still optimizing reward. Results show good performance of the algorithm even in large problems: the most useful parameters of the model are learned quickly and the agent still accumulates high reward throughout the process.
\end{abstract}

\section{Introduction}

Partially Observable Markov Decision Processes (POMDPs) are a popular framework for sequential decision-making in partially observable domains (Littman et al, 1995). Many recent algorithms for efficient planning in POMDPs have been proposed (e.g., Pineau et al. 2003; Poupart \& Boutilier 2005).

However most of these rely crucially on having a known model of the environment. On the other hand, experience-based approaches have been proposed which rely strictly on experimentation with the system to learn a model which can then be used for planning (e.g., McCallum, 1996; Brafman and Shani, 2005; Singh et al. 2004). Yet these typically require very large amounts of data, and are therefore impractical (to date) for large problems. In practice, we would often prefer a more flexible trade-off between these two extremes.

In particular, in many applications it is relatively easy to provide a rough model, though much harder to provide an exact one, and so we would like to use some experimentation to improve our initial model. The overall goal of this work is to investigate POMDP approaches which can combine a partial model of the environment with direct experimentation, in order to produce solutions that are robust to model uncertainty, while scaling to large domains.

We based our work on the idea of active learning (Cohn et al. 1996), a well-known machine learning technique for classification tasks with sparsely labeled data. The goal is to select which examples should be labeled by considering the expected information gain. These ideas extend nicely to dynamical systems such as HMMs (Anderson \& Moore 2005). Applying these ideas to POMDPs, we assume the availability of an oracle that can provide the agent with exact information about the current state, upon request. 
This is a reasonable assumption in a number of real-world POMDP domains. Our work is motivated especially by applications in robotics and dialogue management, where a human is routinely involved in the initial calibration of the robot. However, we assume that using the oracle is expensive and reserved for the learning phase, where we will use it as little as possible.

Our first technique is conceptually simple, though not scalable. In essence, given a problem with model uncertainty, we extend the original problem formulation to include one additional state feature for each uncertain model parameter. The extended model is used for planning, thereby allowing us to obtain a better way of choosing actions, which is also robust to the uncertainty in the model. As discussed in Section 3, this is a straightforward extension of the standard POMDP formulation, which performs well when there are few uncertain parameters but scales poorly.

Our second technique, presented in Section 4, uses oracle queries while the agent interacts optimally with the environment. The query result is used only to improve the model, not in the action selection process. In this framework the uncertainty is represented using a Dirichlet distribution over all possible models, and its parameters are updated whenever new experience is acquired.

\section{Partially Observable Markov Decision Processes}

We assume the standard POMDP formulation (Kaelbling et al., 1998). A POMDP consists of a finite set of states $S$, actions $A$ and observations $Z$. The model is defined by transition probabilities $\left\{P_{s, s^{\prime}}^{a}\right\}=\left\{p\left(s_{t+1}=s^{\prime} \mid s_{t}=s, a_{t}=a\right)\right\}$ and observation probabilities $\left\{O_{s, z}^{a}\right\}=\left\{p\left(z_{t}=z \mid s_{t}=s, a_{t-1}=a\right)\right\}, \forall z \in Z, \forall s, s^{\prime} \in S, \forall a \in A$. It also has a discount factor $\gamma \in(0,1]$ and a reward function $R: S \times A \times S \times Z \rightarrow \mathbb{R}$, such that $R\left(s_{t}, a_{t}, s_{t+1}, z_{t+1}\right)$ is the immediate reward for the corresponding transition.

At each time step, the agent is in an unknown state $s_{t} \in S$. It executes action $a_{t} \in A$, arriving in unknown state $s_{t+1} \in S$ and getting observation $z_{t+1} \in Z$. Agents using POMDP planning algorithms typically keep track of the belief state $b \in \mathbb{R}^{|S|}$, which is a probability distribution over all states given the history experienced so far. A policy is a function that associates an action to each possible belief state. Solving a POMDP means finding the policy that maximizes the expected return $E\left(\sum_{t=1}^{T} \gamma^{t} R\left(s_{t}, a_{t}, s_{t+1}, z_{t+1}\right)\right)$. While finding an exact solution to a POMDP is computationally intractable, many methods exist for finding approximate solutions. In this paper, we use a point-based algorithm (Pineau et al. 2003), in order to compute POMDP solutions. However, other approximations could be used.

We assume the reward function is known, since it is directly linked to the task that the agent should execute, and we focus on learning $\left\{P_{s, s^{\prime}}^{a}\right\}$ and $\left\{O_{s, z}^{a}\right\}$. These probability distributions are typically harder to specify correctly by hand, especially in real applications. For instance in robotics, the sensor noise and motion error are often unknown. We focus on a model-based approach because in many applications the model of the dynamics and observations are re-usable.

To learn the transition and observation models, we assume the agent has the ability to ask a query that will correctly identify the current state (we discuss later how the correctness assumption can be relaxed). This is a strong assumption, but not entirely 
unrealistic. In fact, in many tasks it is possible (but very costly) to have access to the full state information; it usually requires asking a human to label the state. As a result, clearly we want the agent to make as few queries as possible.

\section{Decision-Theoretic Model Learning in POMDPs}

The first algorithm we propose assumes that (1) the parameters of the POMDP model are not known exactly (2) the agent can perform query actions, and (3) these queries are expensive, so they should not be used too much. Based on these three assumptions, we modify the original POMDP model in order to reflect model uncertainty explicitly. First, we increase the number of states: for each uncertain model parameter, we add a new state feature. This feature is typically discretized into $n$ levels. For instance, suppose that for some pair of states $s, s^{\prime} \in S$ and action $a \in A$ we know that $P_{s s^{\prime}}^{a} \in[0.5,1.0]$. We will discretize this interval in $n$ bins and then the state space will receive a new feature, which can take $n$ possible values. We thereby obtain $n$ groups of states; the transitions are such that they always occur between states in the same group. Second, we need to add a "query" action to the set of actions. Finally, we have to set the reward function such that it penalizes query actions adequately.

We analyze the performance of this algorithm on the standard Tiger problem (Littman et al., 1995). We assume that we do not know the probability of the sensor providing the correct state information and consider three possible levels of this probability: 0.7 , 0.8 and 0.9 .

Even with such a simple setting, no exact POMDP solution can be found, but the approximate planning algorithm with a finite horizon finds solutions.

Figure 1 depicts the policies found and the expected reward, as a function of the query penalty. The policies found either alternate between query and the optimal action, or never do any query at all, if the query penalty is too high. Even when no query is done, the agent still manages to learn the observation probabilities. However, we think this is an artifact of having a Listen action, which is in effect a noisy version of a Query action. The fact that some policies use the Listen action but not the Query action suggests that noisy queries may be sufficient to learn the parameters of the system.

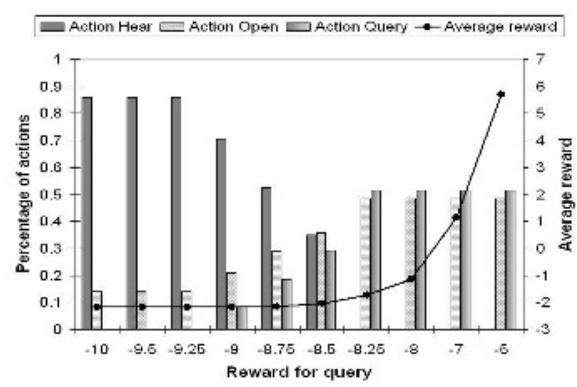

Fig. 1. Experimental results on the Tiger problem using decision-theoretic model learning. The bars indicate the $\%$ of time each action is chosen (during both learning and testing). The line indicates performance of the optimal solution obtained with each reward parameter. 
This approach will not scale well for large POMDPs, because the number of states is multiplied by $n^{k}$ where $n$ is the number of possible values for a given parameter and $k$ is the number of uncertain parameters. This greatly increases the complexity of the belief state and the complexity of the policy. Furthemore, the cost of the query can be very difficult to establish. The results above show that if the cost is too low, the query action is used as part of permanent policies instead of being used only in the beginning to gather information about the model. On the other hand, if the cost is too high, it is likely that the query action will never be picked.

\section{Active Learning in POMDPs}

In the context of POMDPs, transition and observation probabilities are typically specified according to multinomial distributions. Therefore, we now investigate using a Dirichlet distribution to represent the uncertainty over these model parameters (for each state-action pair).

Consider an $N$-dimensional multinomial distribution with parameters $\left(\theta_{1}, \ldots \theta_{N}\right)$. A Dirichlet distribution is a probabilistic distribution over the $\theta$ s, parameterized by hyperparameters $\left(\alpha_{1}, \ldots \alpha_{N}\right)$. The likelihood of the multinomial parameters is defined by:

$p\left(\theta_{1} \ldots \theta_{N} \mid D\right)=\frac{\prod_{i=1}^{N} \theta_{i}^{\alpha_{i}-1}}{Z(D)}$, where $Z(D)=\frac{\prod_{i=1}^{N} \Gamma\left(\alpha_{i}\right)}{\Gamma\left(\sum_{i=1}^{N} \alpha_{i}\right)}$

The maximum likelihood multinomial parameters $\theta_{1}^{*} \ldots \theta_{N}^{*}$ can be computed as:

$\theta_{i}^{*}=\frac{\alpha_{i}}{\sum_{k=1}^{N} \alpha_{k}}, \forall i=1, \ldots N$

The Dirichlet distribution is convenient because its hyper-parameters can be updated directly from data, and we can sample from it using Gamma distributions.

Our algorithm, called MEDUSA for "Markovian Exploration with Decision based on the Use of Sampled models Algorithm" is an active learning approach that follows a familiar scenario. First, the agent samples a number of POMDP models according to the current Dirichlet distribution. The agent takes an action in the environment, and as a result, obtains an observation. At this point, the agent can decide to query the oracle for the true identity of the hidden state. If it does so, it updates the Dirichlet parameters according to the result of the query. This process is repeated until the distribution over models is sufficiently well-known. Table 1 provides a detailed description of these steps, including the implementation details.

A few aspects of this approach are worth discussing further. First, every time a new model is sampled, the agent finds the corresponding (near-)optimal policy, which is then used to select actions. This allows reasonable performance throughout the active learning process: as the sampled models improve, so will the quality of the actions chosen. This also allows the agent to focus the active learning in regions of the state space most often visited by good policies.

We also note that our active learning approach assumes a learning rate, $\lambda$. This is used to update the parameters of the Dirichlet distribution over models following each query. In the experiments, we used a fixed learning rate throughout; however this could be varied (e.g., decreasing over time, as is often done in reinforcement learning). Another important characteristic of our approach is that we need not specify a separate 
Table 1. The MEDUSA algorithm

1. Let $|S|,|Z|$ be the number of states and observations, and $\lambda \in(0,1)$ be the learning rate.

2. Initialize the necessary Dirichlet distributions.

For any unknown transition probability, $T_{s, \cdot}^{a}$, define $\operatorname{Dir} \sim\left\{\alpha_{1}, \ldots \alpha_{|S|}\right\}$.

For any unknown observation $O_{s,}^{a}$, define $\operatorname{Dir} \sim\left\{\alpha_{1}, \ldots \alpha_{|Z|}\right\}$.

3. Sample $n$ POMDPs $P_{1}, \ldots P_{n}$ from these distributions. (We typically use $n=20$ ).

4. Compute the (normalized) probability of each model: $\left\{w_{1}, \ldots w_{n}\right\}$.

5. Solve each model $P_{i} \rightarrow \pi_{i}, i=1, \ldots n$. (We use a finite point-based approximation.)

6. Initialize the history $h=\{\}$

7. Initialize a belief for each model $b_{1}=\ldots=b_{n}=b_{0}$ (We assume a known initial belief $b_{0}$ ).

8. Repeat:

(a) Compute the optimal actions for each model: $a_{1}=\pi_{1}\left(b_{1}\right), \ldots a_{n}=\pi_{n}\left(b_{n}\right)$.

(b) Pick and apply an action to execute: $a_{i}=\pi_{i}\left(b_{i}\right)$ is chosen with probability $w_{i}$.

(c) Receive an observation $z$ and update the history $h=\{h, a, z\}$

(d) Update the belief state for each model: $b_{i}^{\prime}=b_{i}^{a, z}, i=1$..n.

(e) If desired, query the current state, which reveals $s, s^{\prime}$.

(f) Update the Dirichlet parameters according to the query outcome:

$\alpha\left(s, a, s^{\prime}\right) \leftarrow \alpha\left(s, a, s^{\prime}\right)+\lambda$

$\alpha\left(s^{\prime}, a, z\right) \leftarrow \alpha\left(s^{\prime}, a, z\right)+\lambda$

(g) Recompute the POMDP weights: $\left\{w_{1}^{\prime}, \ldots w_{n}^{\prime}\right\}$.

(h) At regular intervals, remove the model $P_{i}$ with the lowest weight and redraw another model $P_{i}^{\prime}$ according to the current Dirichlet distribution. Solve the new model: $P_{i}^{\prime} \rightarrow \pi_{i}^{\prime}$ and update its belief $b_{i}^{\prime}=b_{0}^{h}$, where $b_{0}^{h}$ is the belief resulting when starting in $b_{0}$ and seeing history $h$.

Dirichlet parameter for each unknown POMDP parameter. It is often the case that a small number of hyper-parameters suffice to characterize the model uncertainty.

For example, noise in the sensors may be highly correlated over all states and therefore we could use a single set of hyper-parameters for all states. In this setup, the corresponding hyper-parameter would be updated whenever action $a$ is taken and observation $z$ is received, regardless of the state.

Finally, while Table 1 assumes that a query for the state is performed at every time step, this need not be the case. The decision of when to query could be addressed in a decision-theoretic way (as in section 3), but this is intractable when there are many unknown parameters. In the experimental section below, we investigate various heuristics for deciding when to query. Another approach (which we have not investigated yet) could be to use queries which do not directly reveal the state, but provide related information, since it is possible to update the parameters of the Dirichlet distribution even without explicit state identification.

\section{Experimental Results}

We evaluate MEDUSA first on the standard Tiger problem from Tony Cassandra's repository, considering two cases: (1) The observation probabilities when the Listen action is performed are unknown. (2) All parameters are unknown. 

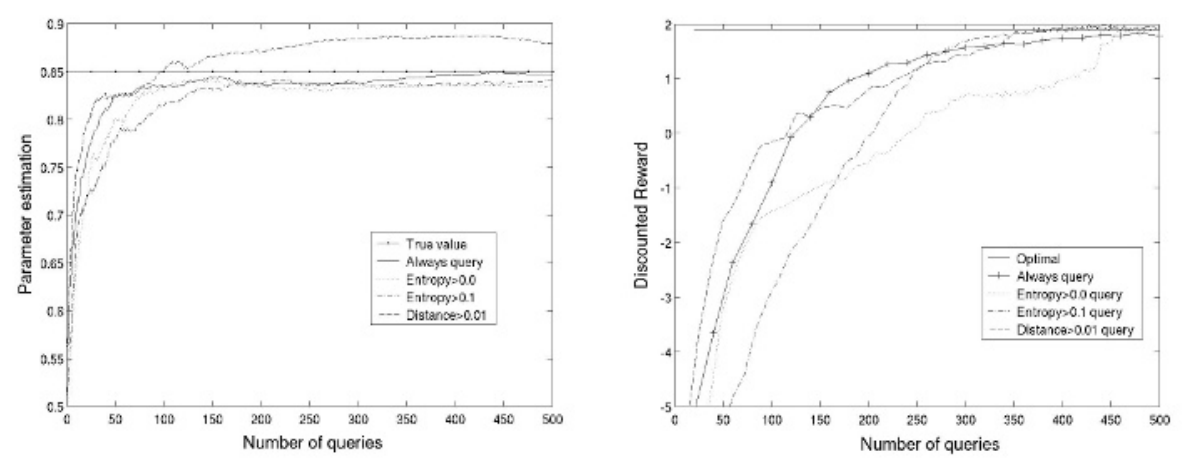

Fig. 2. Experiment 1: Convergence of the estimated parameter $O_{T L, H L}^{\text {Listen }}$ (left). Discounted reward as a function of the number of queries (right).

In the first experiment, we assume that all parameters are set to their correct value, except the $O_{\cdot, \cdot}^{\text {Listen }}$ parameters. (Note that in this experiment the Dirichlet distribution implicitly enforces $O_{s, H L}^{\text {Listen }}=1.0-O_{s, H R}^{\text {Listen }}, \forall s \in S$.) We test the following heuristics for deciding when to query the state:

- Always query: perform a query at every step.

- Entropy>0.0: query when models disagree on which action to select.

- Entropy $>0.1$ : query when the entropy of the probability distribution over actions, as suggested by the different models, is larger than 0.1 .

- Distance $>0.01$ : query when the distance between the belief states corresponding to the different models is too large. This distance is defined by: $\sum_{k=1}^{n} w_{k} \sum_{i \in S}\left(b_{k}(i)-\hat{b}(i)\right)^{2}$, where $\forall i, \hat{b}(i)=\sum_{k=1}^{n} w_{k} b_{k}(i)$

Otherwise, the algorithm is applied exactly as described in Table 1. We also show the return corresponding to the optimal solution obtained when solving the problem with the known parameters.

As shown in Figure 2, the algorithm allows the agent to learn the correct parameters, and performance quickly improves with additional queries. There is no significant difference between the various heuristics for choosing when to perform queries. This suggests that most queries are useful for learning the model.

In the second experiment, all transition and observation probabilities are learned simultaneously. As shown in Figure 3, all correct parameters are learned accurately with few queries (200-300), and about 2000 queries are needed to reach the optimal reward. In comparison, recent results for learning models use on the order of $10^{6}-10^{7}$ steps to learn this problem and others of similar size(Singh et al. 2004). We note, however, that these algorithms are not allowed to query an oracle for additional information, and therefore face a harder problem.

We also tested the scaling of the algorithm on a larger domain called Tiger-Grid (Littman et al. 1995). It has 36 states, 5 actions, 17 observations, for a total of $9000+$ parameters. It also features probability distributions that are more characteristic of real robots, including noisy motion and sensors. In a domain of this size, it is unlikely that all parameters will be uncorrelated. More likely, there are a few effects (e.g., sensor noise) 

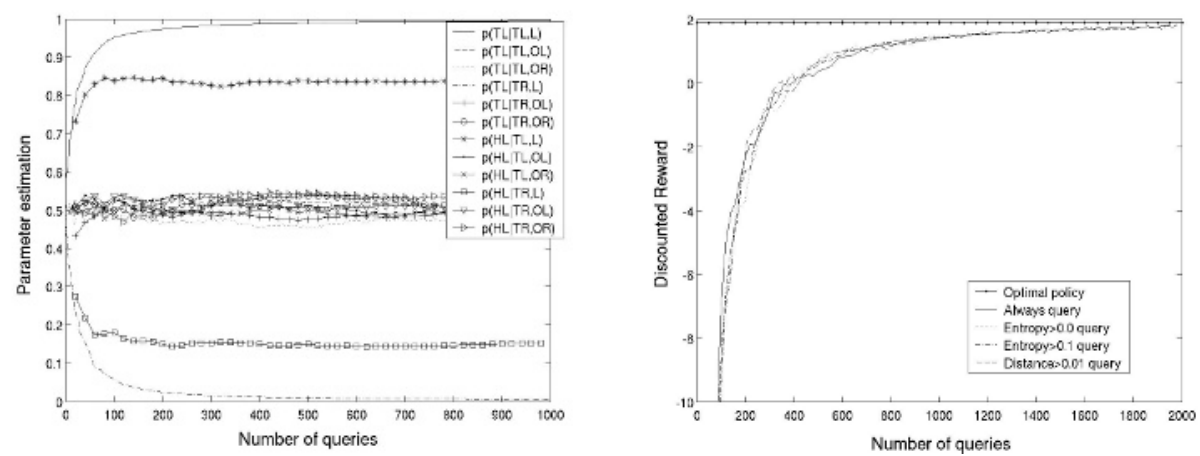

Fig. 3. Experiment 2: Convergence of the parameters for the "always-query" case (left). Discounted reward as a function of the number of queries (right).

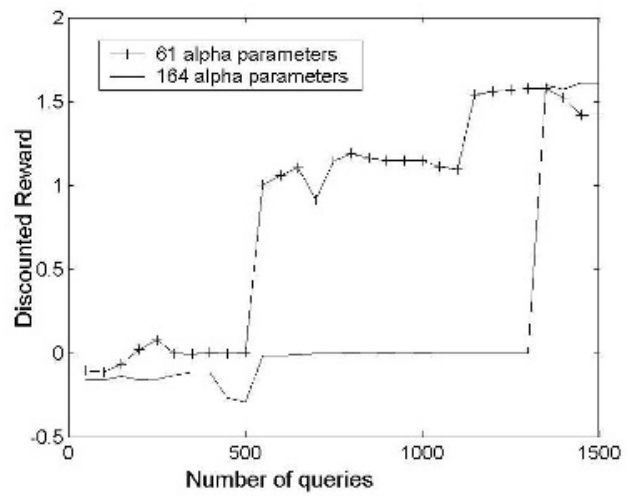

Fig. 4. Experiment 3: Discounted reward as a function of the number of queries for Tiger-grid domain for different numbers of alpha parameters .

that are similar over a number of states. Therefore, the uncertainty in these parameters can be correlated through a single hyper-parameter, rather than learning all parameters independently. In the experiments, we apply the algorithm described in Table 1 and vary the number of $\alpha$ parameters used. The results in Figure 4 confirm that with the appropriate number of parameters, the algorithm can effectively improve the model using queries. As expected, the speed of learning depends on the number of hyper-parameters. Thus, our approach can effectively trade-off learning speed versus model accuracy.

\section{Discussion}

Chrisman (1992) was among the first to propose a method for acquiring a POMDP model from data. Shatkay \& Kaelbling (1997) used a version of the Baum-Welch algorithm to learn POMDP models for robot navigation. Bayesian exploration was proposed by Dearden et al.(1999) to learn the parameters of an MDP. Their idea was to reason 
about model uncertainty using Dirichlet distributions over uncertain model parameters. The initial Dirichlet parameters can capture the rough model, and they can also be easily updated to reflect experience. The algorithm we present in Section 4 can be viewed as an extension of this work to the POMDP framework, though it is different in many respects, including the handling of exploration vs exploitation.

Recent work by Anderson and Moore (2005) examines the question of active learning in HMMs. In particular, their framework for model learning addresses a very similar problem (albeit without the complication of actions). The solution they propose selects queries to minimize error loss (i.e., loss of reward). However, their work is not directly applicable since they are concerned with picking the best query for a large set of possible queries. In our framework, there is only one query to consider, which reveals the current state.

Finally, our work resembles some of the recent approaches for handling model-free POMDPs (McCallum, 1996; Brafman \& Shani, 2005; Singh et al. 2004). Unlike these approaches, we make here strong assumptions about the existence of an underlying state which allows us to partly specify a model whenever possible, thereby making the learning problem much more tractable (e.g., orders of magnitude fewer examples). The other key assumption we make, which is not used in model-free approaches, regards the existence of an oracle (or human) for correctly identifying the state following each query. We are currently studying how this assumption can be relaxed.

The question of when to do queries, and whether to consider a more varied set of queries is of interest. Clearly the decision-theoretic approach of Section 3 can contribute to this decision. However, using this approach calls for POMDP algorithms that can handle very large (possibly continuous) state spaces, and these are currently lacking. MEDUSA has a very good performance and scales nicely, but a theoretical analysis of its convergence properties remains to be done. Our next goal is to apply MEDUSA to the control of a mobile interactive robot.

\section{References}

Anderson, B. and Moore, A. "Active Learning in HMMs". ICML 2005.

Brafman, R. I. and Shani, G. "Resolving perceptual asliasing with noisy sensors". NIPS 2005.

Cohn, D. A., Ghahramani, Z. and Jordan, M. I. "Active Learning with Statistical Models". NIPS 1996.

Dearden, R.,Friedman, N.,Andre, N., ”Model Based Bayesian Exploration”. UAI 1999.

Kaelbling, L., Littman, M. and Cassandra, A. "Planning and Acting in Partially Observable Stochastic Domains" Artificial Intelligence. vol.101. 1998.

Littman, M., Cassandra, A., and Kaelbling,L. "Learning policies for partially observable environments: Scaling up", Technical Report. Brown University, 1995.

McCallum, A. K. Reinforcement Learning with Selective Perception and Hidden State. Ph.D. Thesis. University of Rochester. 1996.

Pineau, J., Gordon, G. and Thrun, S. "Point-based value iteration: An anytime algorithm for POMDPs". IJCAI. 2003.

Poupart, P. and Boutilier, C. "VDCBPI: an Approximate Scalable Algorithm for Large Scale POMDPs". NIPS 2005.

Singh, S., Littman, M., Jong, N. K., Pardoe, D., and Stone, P. "Learning Predictive State Representations". ICML 2003. 